\title{
Large saddle embolus: an unusual cause of collapse
}

\begin{abstract}
Background: Pulmonary embolisms are a relatively common and potentially lifethreatening cardiovascular disorder. Diagnosis can be difficult due to variation in presentation, therefore suspicion should always be high for a venous thromboembolic cause for any unusual presentations, such as an unprovoked collapse. Treatment for pulmonary embolisms remains somewhat conflicted, particularly in cases with complex comorbidities.
\end{abstract}

Case presentation: A 69year old Caucasian male was admitted with a new history of multiple collapses over the past three days. He had had a small GI bleed a month ago, which was being managed with proton pump inhibitors. A CTPA was performed, and showed a large saddle thrombus. Despite the radiological diagnosis, the patient was haemodynamically stable, so he was not thrombolysed. Considering his recent history of a GI bleed, he was started on Warfarin due to its reversibility. Previous studies have suggested that the incidence of bleeding while being anticoagulated depends on the site of bleeding and the time elapsed between bleeding and thrombotic event. It has been suggested previously that the benefits of anticoagulation therapy outweigh the risks for those with GI bleeds. The patient was loaded on Warfarin and was discharged without complication.

Conclusion: This case demonstrates an unusual presentation of a pulmonary embolism. The corresponding radiological image shows a very significant saddle embolus, which shows how the acuity of the clinical presentation may not match the severity of the pathology seen radiologically. In addition, the treatment used for the patient was somewhat equivocal due to the patient's recent history of a small upper GI bleed, resulting in Warfarin being prescribed. Recent evidence has been unclear and conflicting about what management option is best for managing venous thromboembolisms on a background of GI bleeding. This case highlights the need for further RCTs comparing management and clearer guidelines in regards to anticoagulation.

Keywords: pulmonary medicine, pulmonary embolism, gastrointestinal bleeding, anticoagulation, case report, condition, hemoptysis, thromboembolic, pulmonary, palpitations
Volume 2 Issue I - 2017

Wong Cynthia

Diana Princess of Wales Hospital, UK

Correspondence: Wong Cynthia, Diana Princess of Wales Hospital, Scartho Road, Grimsby DN33 3RB, UK, Email cindymwong@gmail.com

Received: October 29, 2016 | Published: February 03, 2017
Abbreviations: GI, gastrointestinal; NOAC, new oral anti-coagulant; CTPA, computed tomography pulmonary angiogram; $\mathrm{RCT}$, randomized control trial

\section{Introduction}

Pulmonary embolisms are a relatively common cardiovascular disorder, with approximately 1.83 per thousand per year, although the real number of cases may be higher due to the number of silent pulmonary embolisms. ${ }^{1}$ It can be a life-threatening condition, particularly if not diagnosed appropriately. One difficulty in diagnosing a pulmonary embolism is the variation in presentation, which can involve shortness of breath, cough (with or without hemoptysis), chest pain, collapse, fever, palpitations, or no symptoms at all. Although smaller clots may present asymptomatically, it may be possible for larger pulmonary embolisms to present relatively innocuously as well. Therefore, suspicion should always be high for a venous thromboembolic cause for an unusual presentation, such as an unprovoked collapse. The treatment for pulmonary embolisms remains conflicted, particularly in cases with complex comorbidities.

\section{Case presentation}

A 69year old Caucasian male was admitted with a new history of multiple collapses over the past three days. He had no palpitations and did not lose consciousness during these collapses. He described a mild shortness of breath while at rest, but no chest pain, cough, or haemoptysis.

His past medical history was significant only for a small GI bleed a month ago, which was being managed with proton pump inhibitors. He was taking no other medications. He had no significant family history. He was a non-smoker and non-drinker. On examination, he was found to be clinically well, with all observations within normal ranges. His heart sounds were normal, and chest was clear to auscultation. However, because of the short history of shortness of breath and multiple collapses, a CT pulmonary angiogram was performed. The CT pulmonary angiogram was helpful to visualise the pulmonary arteries by injecting intravenous contrast and showed a large saddle thrombus where the thrombus causes a mass filling defect and appears dark in the pulmonary bifurcation (Figure 1). Despite the radiological diagnosis, the patient was haemodynamically stable, so thrombolysis was deemed unnecessary, as it is not indicated in a nonmassive pulmonary embolism. ${ }^{2}$ His clinical condition was closely monitored for any deterioration.

There was some confusion as to what anticoagulant to use for his saddle embolus. Rivaroxaban, a newer oral anticoagulant (NOAC), is commonly used for pulmonary embolisms at this district hospital due to the advantage of its more fixed anticoagulant effect compared 
to warfarin. The EINSTEIN-PE study suggested a noninferiority of Rivaroxaban compared with the previous treatment standard of vitamin $\mathrm{K}$ anatagonists such as warfarin. ${ }^{3}$ However, after discussion with the consultant haematologist, this patient was started on warfarin, due to its reversibility in regards to his recent history of a small GI bleed. The patient was loaded on Warfarin while being bridged with low molecular weight heparin as per guideline recommendations and was discharged when the INR was within range without complication. ${ }^{2}$

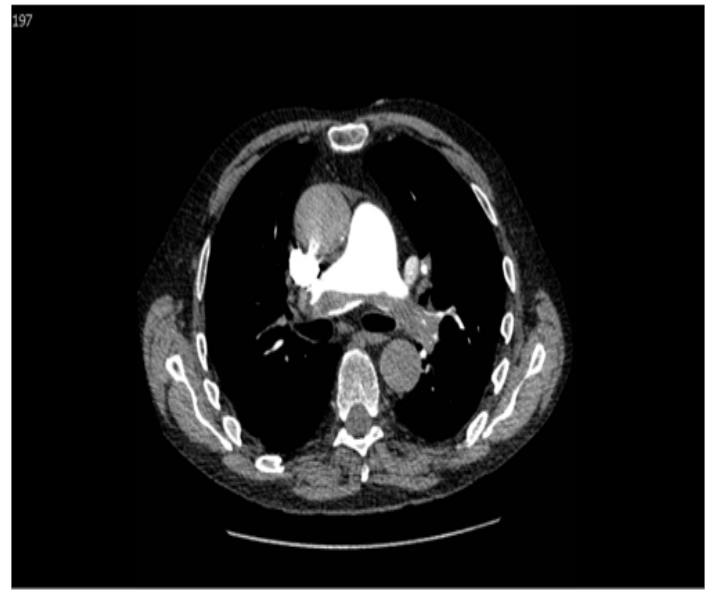

Figure I Large saddle thrombus demonstrated in the pulmonary artery bifurcation.

\section{Discussion}

Previous studies have suggested that the incidence of bleeding while being anticoagulated depends on the site of bleeding (with GI tract bleeds conferring an increased likelihood of re-bleeding) and the time elapsed between bleeding and thrombotic event. ${ }^{4} \mathrm{~A}$ bleed 14days ago is classically considered to be a contraindication to anticoagulation, so in the case of this patient's bleed four weeks ago, anticoagulation should not be necessarily contraindicated. ${ }^{4}$ It has been suggested previously that the benefits of anticoagulation therapy outweigh the risks for those with GI bleeds. ${ }^{5}$ Therefore, in cases such as this one, the need for some form of anticoagulation outweighs the risk of causing rebleeding, so it was appropriate to consider anticoagulation.

Further difficulty in clinical decision making arises when trying to determine which anticoagulant is most appropriate. With the introduction of NOACs such as Rivaroxaban, the burden of choice has become even greater. NOACs have the advantage of more predictable anticoagulant effect at fixed doses compared to the narrow therapeutic range of warfarin. NOACs also carry the disadvantage of having no readily available reversal agent, compared with warfarin, where vitamin $\mathrm{K}$ can be given to promote the synthesis of new clotting factors, or fresh frozen plasma can be given when urgent reversal is required. Both NOACs and Warfarin carry the risk of bleeding, with current evidence being conflicted on which carries a higher risk of bleeding.

One meta-analysis by Holster et al suggested that NOACs conferred a higher risk of GI bleed when compared to the standard treatment of warfarin. ${ }^{6}$ Alternatively, a more recent meta-analysis by Caldeira et al., ${ }^{7}$ suggested that NOACs had a lower case fatality due to major bleeding when compared to warfarin, though this decreased risk was particularly seen in atrial fibrillation patients, and was less pronounced in patients with venous thromboembolisms. Both meta-analyses compared Rivaroxaban, Apixaban, Dabigatran, and Edoxaban for the indications of atrial fibrillation and venous thromboembolism. The cause for the difference in results of these meta-analyses may be due to a variety of reasons including varied definitions of "major" bleed. The study by Holster et al also specified that the risk of GI bleeding was increased, while the study by Caldeira et al., ${ }^{7}$ was focused on bleeding related mortality, and was less specifically about GI bleeding. These discrepancies make it difficult to directly compare the studies, however the lack of clear guidance highlights how challenging it can be to make informed decisions in clinical practice. Further studies may be beneficial, such as RCTs with more specific outcomes comparing the risk of GI bleeding while taking NOACs compared with those taking vitamin $\mathrm{K}$ anatagonists in patients who have had previous GI bleeds. More specific studies can help to inform decision making in more complex cases.

\section{Conclusion}

Pulmonary embolisms can be difficult to diagnose due to variations in clinical presentation, and a high suspicion for a pulmonary embolism should always be maintained, especially when the size of the clot may not necessarily correspond with the clinical picture. As an increasing number of patients are put on anticoagulant agents and suffer thromboembolic events, we need to be aware and informed about how to deal with increasingly complex cases. A paucity of evidence exists regarding the appropriate management of venous thromboembolisms on a background of GI bleeding. This case report suggests that further RCTs are required to compare the use of different anticoagulant agents, particularly in cases involving previous bleeds.

\section{Consent}

Written informed consent was obtained from the patient for publication of this case report and any accompanying images. A copy of the written consent is available for review by the Editor-in-Chief of this Journal.

\section{Author's contributions}

The author was involved the management of the patient's condition, discussed the case with the consultant haematologist, conceived of the case report, and wrote the manuscript.

\section{Acknowledgements}

I would like to thank the patient for providing his medical record and imaging to publish this medical report, as well as the doctors at the Diana Princess of Wales Hospital for their help and support.

\section{Conflict of interest}

Author declares that there is no conflict of interest.

\section{References}

1. Oger E. Incidence of venous thromboembolism: a community-based study in Western France. Thromb Haemost. 2000;83(5):657-660.

2. British Thoracic Society guidelines for the management of suspected acute pulmonary embolism. Thorax. 2003;58:470-483.

3. Einstein PE Investigators, Buller HR, Prins MH, et al. Oral rivaroxaban for the treatment of symptomatic pulmonary embolism. $N$ Engl J Med. 2012;366(14):1287-1297. 
4. Nieto JA, Bruscas MJ, Ruiz-Ribo D, et al. Acute venous thromboembolism in patients with recent major bleeding. The influence of the site of bleeding and the time elapsed on outcome. J Thromb Haemost. 2006;4(11):2367-2372.

5. Witt DM, Delate T, Garcia DA, et al. Risk of thromboembolism, recurrent hemorrhage, and death after warfarin therapy interruption for gastrointestinal tract bleeding. Arch Intern Med. 2012;172(19):1484-1491.
6. Holster IL, Valkoff VE, Kuipers EJ, et al. New oral anticoagulants increase risk for gastrointestinal bleeding: a systematic review and meta-analysis. Gastroenterology. 2013;145(1):105-112.

7. Caldeira D, Rodrigues FB, Barra M, et al. Non-vitamin K antagonist oral anticoagulants and major bleeding-related fatality in patients with atrial fibrillation and venous thromboembolism: a systematic review and meta-analysis. Heart. 2015;101(15):1204-1211. 